\title{
Application of the Information Security Services Platform Cloud
}

\author{
Deng Ping \\ Beijing Institute of Technology, Beijing 100081, China \\ Guangzhou Polytechnic of Sports, Guangzhou 510650, Guangdong, China \\ Information Engineering Institute \\ cityping@163.com
}

\begin{abstract}
Compared with the traditional software architecture, cloud computing has the advantages of low cost, rapid deployment, flexible, and so on, in the enterprise to use cloud computing can greatly improve the enterprise IT resource management, improve the operational strategy and reduce the operational costs of the enterprise. With the study of the various companies to cloud computing technology and applications, cloud computing data security has become a key factor in the development of cloud computing and bottlenecks. In this paper, we introduce the concept and knowledge of cloud computing, and give a solution of secure data storage based on cloud computing.
\end{abstract}

Key words: Cloud Computing; Secure Storage; Storage Service

\section{INTRODUCTION}

With the rapid development of information technology, the demand for data storage and storage has been increased, and the traditional model of high performance server to meet the needs of users has greatly increased the waste of resources. Cloud computing (Computing Cloud) is a new computing model which is adapted to the network service from the high concentration, high cost, low cost, high distribution, low cost, high general purpose. It is a new research and application field, which is increasingly concerned by the relevant enterprises and research institutions.

Cloud computing is a computing based on the Internet, through this way, sharing of software and hardware resources and information, can be required to provide to the computer and other equipment. At present, the cloud computing in many enterprise users to provide high performance computing is playing its low cost, rapid deployment, flexible adjustment scale and other advantages. With the rapid development of cloud computing, more and more commercial data are stored in different networks. How to make sure that the data is safe and low in the network, the confidentiality and integrity of data is the key point of the research and application of cloud computing. The security of data has become the key factor and bottleneck of the development of cloud computing. This paper introduces the related concepts and knowledge of cloud computing, and presents a security data storage model based on cloud computing and service oriented, which provides a strong guarantee for enterprise data secure transmission and storage.

\section{RELATED KNOWLEDGE}

\section{A. Cloud computing service model}

The application of cloud computing to information technology is huge, which covers all aspects of the application of the underlying infrastructure to the upper level, including the server, network transmission, data storage, data security and system management, and so on. Cloud computing is not only a new technology, but also a new business model; it spawned a large number of new industrial applications and business growth point.

At present, the service mode of cloud computing is mainly divided into three kinds: namely, the software is the service, the platform is the service, and the infrastructure is the service. 
Software-as-a-Service (Software, SaaS), is based on the Internet to provide software services software applications, is a new software application mode, is the latest trend in software development. This type of cloud computing through the browser to upload software to the network user, the user saves the server and software licensing expenses; for software providers, only need to maintain a software program is sufficient to reduce the cost of software maintenance. Compared to the traditional service mode, SaaS has the following advantages:

SaaS mode to reduce the cost of traditional software licensing;

Software providers will be deployed on the unified server software, to avoid the user server hardware, security equipment and software upgrades and maintenance of spending;

A large number of new technologies, such as WebService, provide a simpler, more flexible and more practical SaaS;

The user can accord the actual needs of on-demand ordering software application service.

Platform that is service (Platform-as-a-Service), Platform as a service (PaaS) is a software development platform as a service provided by the business model. PaaS is a specific application of SaaS, the service provided in SaaS is the software, while the service provided in PaaS is the platform. The emergence of PaaS can accelerate the development of SaaS, especially to speed up the development of SaaS application. PaaS has the following three aspects:

The most fundamental difference between PaaS and other services provided by PaaS is that the provides a basic platform, rather than some kind of application, which is built and operated by a dedicated platform service provider;

PaaS operators need to provide services, not just a simple platform, but also for the platform for technical support services, and even for the platform and the application system development, optimization and other services;
PaaS operators are different from other services; the service is a strong and stable operation platform, as well as professional technical support team.

Infrastructure-as-a-Service, IaaS ,Infrastructure is a service (IaaS), which provides consumers with the use of infrastructure, including processing, storage, network and other basic computing resources, users can deploy and run arbitrary software, including: operating systems and applications. The consumer does not manage or control any cloud computing infrastructure, but it can control the selection of operating systems, storage space, and deployment of applications, and it is possible to obtain control of the network components (e.g., firewalls, load balancing, etc.). At present, the commonly used IaaS platforms are: OPEN Stack and eucalyptus.

\section{B. Architecture of cloud storage}

Cloud storage is the basis and core of cloud computing, which means that a large number of different types of storage devices through the application of the application software can be used to work together to provide a system for data storage and business visit. Users use cloud storage, not the use of a storage device, but the use of a data access service to the entire cloud storage system. The core of cloud storage is the combination of application software and storage device, through the application of software to achieve the storage device to the storage service changes. The structure model of cloud storage is shown in Figure 1, from top to bottom, the access layer, application interface layer, basic management layer, storage layer.

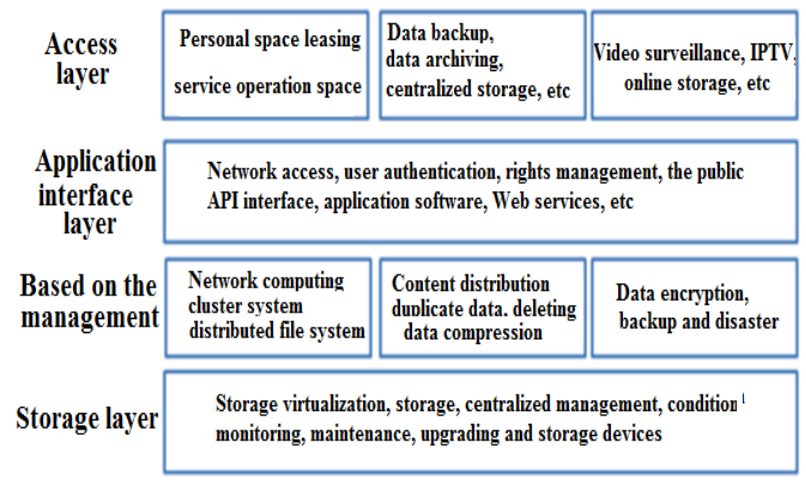

Fig.1 Cloud storage structure model 
Access layer: provides the application interface of the login cloud storage system, and provides different access types and access methods according to different client units.

Application interface layer: the most flexible part of the cloud storage. Each cloud storage and operation unit according to different business types, the development of different service interfaces and provide different application services.

Basic management: through clustering, distributed file systems and other technologies to achieve a collaborative work between multiple storage devices, so as to mention the greater and stronger data access to the east.

Storage layer: the function of the logic virtualization management, multi-link redundancy management, and the state monitoring and fault maintenance of the storage device.

\section{DESIGN OF SECURE DATA STORAGE SYSTEM BASED ON CLOUd COMPUTING}

The security data storage system based on cloud computing includes four parts, that is, storage cloud, control center, user interface, client. Its architecture is shown in figure 2.

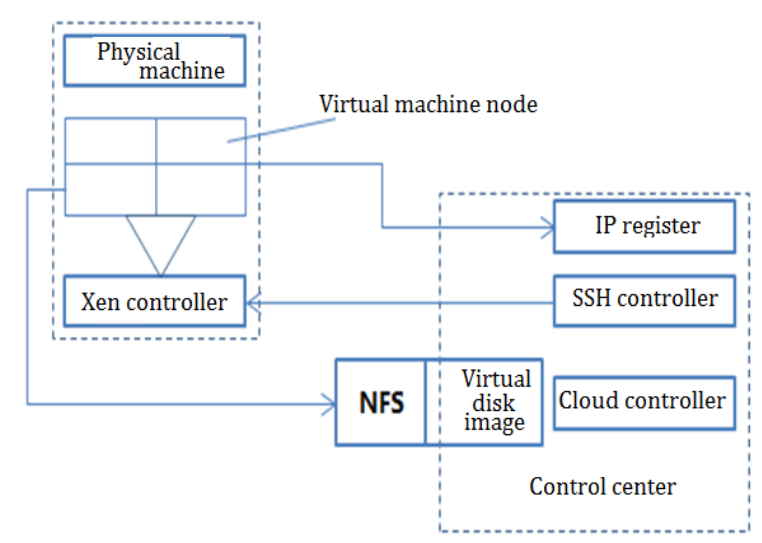

Fig.2 Security data storage system architecture based on Cloud Computing

\section{A. Storage Cloud}

Storage Cloud includes public data storage and private data storage cloud two parts, storage cloud is responsible for saving all the data and operation of the user, which itself does not need to provide computing capabilities, private data storage cloud for enterprise internal requirements to achieve a secure data storage platform. The division of public data storage and private data storage cloud saves the storage space, and also solves the user's concerns about the security of the platform.

The implementation of private data storage cloud is: the physical machine in the cluster is installed with Linux Xeon system, and the various physical machines are turned on the SSH service and the control center is realized by SSH and $\mathrm{XM}$ in Xeon. Logical connections between the components of the private cloud are shown in figure 3 .

\section{B. Control center}

The control center is responsible for data processing and calculation of the whole system, including: data encryption and decryption, data compression, data indexing, and so on.

\section{User interface}

User interface for different users to achieve different service interfaces, including user data format conversion, user rights management and user authentication and other functions.

\section{Client}

The client is the end user of the data. The client sends service request through the node server. The control center receives the service request and processes it, and returns the service data to the client. Among them, the data between the client and the control center, the data between the control center and the storage cloud are transmitted in the form of cipher text.

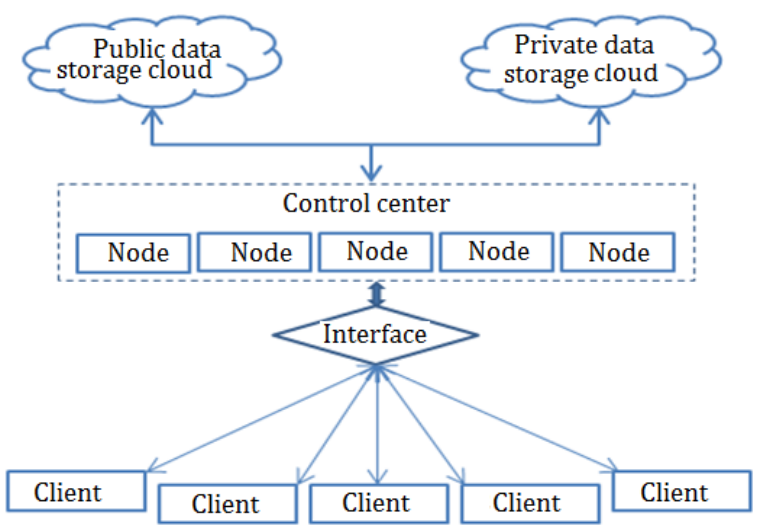

Figure. 3 Logical Diagram of the Components of the Private Cloud 


\section{SUMMARY}

Cloud computing has high performance, low cost and flexibility. The rapid development of cloud computing makes it more and more important. In this paper, we propose a secure data storage model based on cloud computing.

\section{CONFLICT OF INTEREST}

The authors confirm that this article content has no conflicts of interest.

\section{ACKNOWLEDGMENT}

This work was supported by the Guangdong natural fund team research project, China (no.S2012030006242), National Natural Science Foundation of China (no.61272067.

\section{REFERENCES}

[1] Cai Yingfang; the security risk assessment model of information system based on cloud computing [J]; China management information; 122010

[2] Shao Zongyou; Zhang Xiang; Bai Xiujie; Yuan Wei; Liu Xinchun;; [J];; information security and technology; information security and technology; 9

[3] Zhou Yinghong; the status quo and development of information security technology assessment [J]; computer security; 200911

[4] Chang Quan Wang; AI atmosphere; Yao Jianwen; cloud computing environment of digital library information resources security strategy [J]; Journal of information; 201003

[5] Hu Chunhui; cloud computing security risks and protection technology framework analysis [J]; information network security; 2012(7)

[6] Liu Yunlong; Zeng Jin; FA Wang Liu; levy; safety risk oriented cloud computing test technology $[\mathrm{J}]$; microelectronics and computer; 2013( 06)

[7] Zhang Yahong; Zheng Lihua; Zou Guoxia; information security in the cloud computing environment $[\mathrm{J}]$; network security technology and application; 2010(10)

[8] Ying;; cloud computing and [J] security issues; enterprise herald ; 2009(10)

[9] leaves a; Zhang gongrang; cloud computing and information security; [J]; value engineering; 2011(1)

[10] Guo Chunmei; Bi Xueyao; Yang Fan; cloud computing security technology research and trend [J]; information network security; 2010(4)

[11] Xu Gang;; cloud computing and cloud security [J]; information security and technology; 2011 (Z1)

[12]Chen Honghui; cloud computing security challenges [J]; computer knowledge and technology; 2011(24) 